\title{
MicroRNAs and gene regulatory networks: managing the impact of noise in biological systems
}

\author{
Héctor Herranz ${ }^{1}$ and Stephen M. Cohen ${ }^{1,2,3}$ \\ ${ }^{1}$ Institute of Molecular and Cell Biology, Singapore 138673; ${ }^{2}$ Department of Biological Sciences, National University of \\ Singapore, Singapore 117604
}

Biological systems are continuously challenged by an environment that is variable. Yet, a key feature of developmental and physiological processes is their remarkable stability. This review considers how microRNAs contribute to gene regulatory networks that confer robustness.

The molecular processes that drive cellular functions are inherently noisy. Intrinsic and extrinsic factors can affect levels of gene expression, protein stability, protein modification, enzymatic reactions rates, and signal transduction processes. The ways in which cells and organisms use noise and deal with its impact have become the focus of considerable interest (for review, see Raser and $\mathrm{O}^{\prime}$ Shea 2005; Alon 2007; Barkai and Shilo 2007; Losick and Desplan 2008). This review considers some of the ways in which microRNAs (miRNAs) contribute to these processes. It focuses on the roles of miRNAs in small-scale gene regulatory networks with defined topology, with emphasis on the relationship between miRNAs and noise. The biological properties of miRNAs have been reviewed extensively in recent years, so we assume that the reader is familiar with what miRNAs are and how they work (for reviews, see Bushati and Cohen 2007; Eulalio et al. 2008; Stefani and Slack 2008; Bartel 2009).

Some biological processes involve stochastic decisions, which make use of noise. Examples include the switch between growth and competence phases in Bacillus subtilis, and the choice of Rhodopsin type during photoreceptor differentiation in the Drosophila eye (Losick and Desplan 2008). In other contexts, noisy gene expression has the potential to be harmful, as is illustrated in some of the examples that follow. Gene regulatory networks involving positive and negative feedback have the potential to control the effects of noise, by buffering its impact on gene expression (e.g., Lee et al. 2002; Milo et al. 2002). Computational analysis has suggested that miRNAs are overrepresented in gene regulatory networks, suggesting

[Keywords: MicroRNAs; noise; robustness; regulatory networks] ${ }^{3}$ Corresponding author.

E-MAIL scohen@imcb.a-star.edu.sg; FAX 65-6872-7089.

Article is online at http://www.genesdev.org/cgi/doi/10.1101/gad.1937010. that they confer useful regulatory possibilities (Tsang et al. 2007; Martinez et al. 2008).

Complex networks often include a small set of recurring circuits called network motifs (for review, see Alon 2007). These motifs are found more often than would be expected by chance in gene regulatory networks, from bacteria and yeast to plants and animals. Network motifs can be subdivided into two broad categories: feedback and feedforward. The utility of positive and negative feedback regulation is intuitively obvious (Fig. 1A). For example, a transcription factor can limit its own activity by inducing a repressor. Alternately, a positive feedback loop can produce a robust developmental switch by ensuring an "all-or-none" outcome. Feedforward motifs are less intuitive, and are based on regulators that act both directly and indirectly on their downstream targets (Fig. 2A). In the following sections, we consider the roles that miRNAs play in feedback and feedforward networks.

\section{Feedback motifs as switches}

\section{Using noise}

Evidence is beginning to emerge that miRNAs can be involved in the mechanisms by which noise is used to trigger stochastic developmental decisions. Sense organ specification in the Drosophila peripheral nervous system involves a key element of stochasticity. Sense organ primordia are initially defined as small groups of cells that express a set of "proneural" basic helix-loop-helix (bHLH) transcription factors of the Hairy-E(spl), or HES, family. Definition of the proneural cluster at this level is deterministic, based on programmed control of gene expression, but the choice of which cell will become the sense organ precursor (SOP) is stochastic. One cell is selected by chance, as a consequence of fluctuation in the level of the transcription factor Senseless (Nolo et al. 2000). Higher Senseless levels lead to an increase in proneural gene expression in the SOP, which feeds back to increase Senseless expression (Fig. 1B). As well, Senseless increases expression of the Notch ligand Delta, which in turn leads to increased Notch signaling activity in the adjacent cells (Fig. 1B). Notch activity represses 
A


B

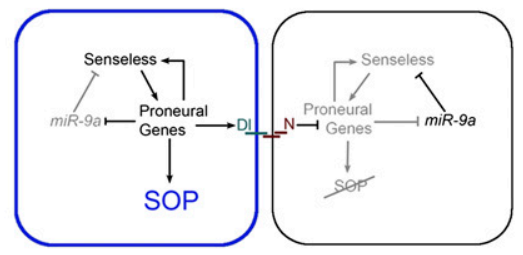

$\mathrm{E}$

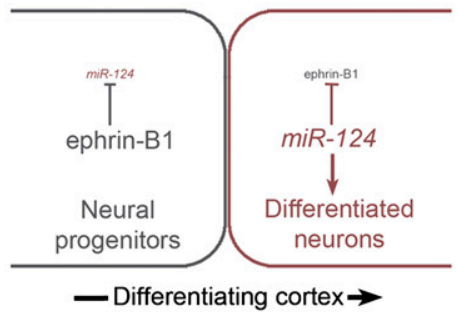

C
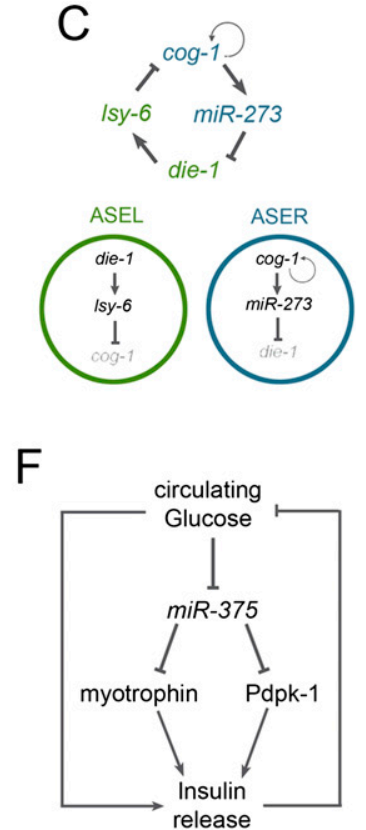

Figure 1. miRNAs involved in feedback motifs. $(A)$ Simple positive and negative feedback loops. $(B)$ miR-9a in a positive feedback loop in SOP specification in Drosophila. The boxes represent cells within the proneural cluster. In the blue cell, the Senseless-proneural gene positive feedback circuit dominates and leads to SOP differentiation. Senseless activates Delta, increasing Notch activity in adjacent cells. Notch inhibits proneural genes, allowing $m i R-9 a$ repression of Senseless to dominate in these cells. $(C) 1 s y-6$ and $m i R$ 273 in a negative feedback loop controlling left/right asymmetry in ASE neurons of C. elegans. The transcription factors encoded by cog-1 and die-1 are active in ASER and ASEL, respectively. cog-1 activates miR-273 miRNAs, which repress die-1 in ASER. die-1 activates 1 sy-6, which represses $\operatorname{cog}-1$ in ASEL. This initial asymmetry is stabilized by positive autoregulation of cog-1 in ASER. $(D)$ miR-14 acts in a negative feedback loop modulating EcR activity. EcR positively autoregulates. miR-14 limits EcR levels, which in turn negatively regulates $m i R-14$. $(E)$ miR-124 in a negative feedback loop in the mouse cortex. The open boxes represent cells. Ephrin-B1 activity represses $m i R-124$ in neural progenitors. $m i R-124$ inhibits Ephrin-B1 and induces neuronal differentiation. $(F) m i R-375$ acts in a negative feedback regulating insulin secretion. High glucose levels inhibit miR-375. miR-375 inhibits myotrophin and Pdpk-1, reducing insulin secretion. Circulating glucose promotes insulin secretion directly, and indirectly represses miR-375.

proneural gene expression in these cells, and reinforces the advantage of the Senseless- and Delta-expressing cell toward becoming the SOP (Jafar-Nejad et al. 2003). This system combines positive feedback with lateral inhibition to select a single cell and promote its differentiation into the SOP. However, more recent studies indicate this circuit is not enough to ensure a reliable outcome ( $\mathrm{Li}$ et al. 2006; for review, see Cohen et al. 2006). Flies lacking miR-9a sporadically produce extra sense organs from individual proneural clusters. This indicates that more than one cell in the cluster can cross the threshold level of Senseless activity required to trigger the SOP switch. Feedback regulation is an essential element of the mechanisms that transform noise into switches (for review, see Losick and Desplan 2008), but positive feedback loops are inherently labile. Additional mechanisms are needed to ensure that random fluctuation in transcription factor levels does trigger positive feedback inappropriately. In this biological context, the presence of miR-9a sets a threshold that Senseless transcription must overcome in order to trigger the Senseless-proneural feedback loop (Fig. 1B).

An important feature of this circuit is that miR-9a expression is under proneural gene control. miR-9a levels are initially uniform in the proneural cluster, but as
Senseless activity increases proneural gene expression in the SOP, miR-9a levels are reduced. miR-9a levels remain high in the surrounding cells, where proneural gene activity is kept low through Notch activity. The opposing regulation of Senseless and miR-9a is an integral element of the switch. The outcome is a situation in which initially overlapping expression domains resolve into spatially reciprocal domains of the miRNA, and its target, Reciprocity, is a common feature of miRNA and target expression (Stark et al. 2005) and serves to reinforce the differences between the cell types. The transcriptional feedback system is triggered by random fluctuations in gene expression, and the miRNA helps to reinforce selection of only one peak of Senseless expression while filtering out others that might have been able to surpass the threshold in the absence of the miRNA. Thus, miR-9a contributes to the robustness of the noise-dependent switch.

\section{lsy-6 and miR-273 contribute to a bistable switch}

The nematode Caenorhabditis elegans makes a pair of chemosensory neurons that are functionally asymmetric on the left and right sides of the animal. Work from the Hobert laboratory (for review see Hobert 2006) has shown 
A

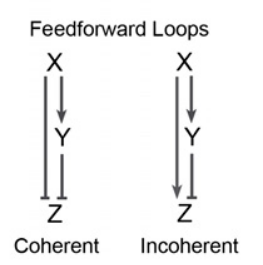

B

C

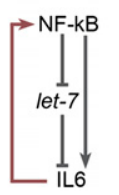

$\mathrm{D}$

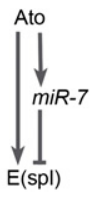

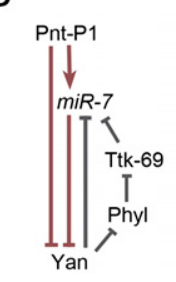

E



Figure 2. miRNAs involved in feedforward motifs. $(A)$ Feedforward motifs where $\mathrm{X}$ regulates $\mathrm{Z}$ directly and indirectly through regulation of $Y$. Coherent motifs have the direct and indirect paths from $\mathrm{X}$ acting on the target $\mathrm{Z}$ in the same direction. Incoherent motifs have opposite signs for the two paths. $(B)$ $m i R-7$ acts in two coherent feedforward motifs. Yan is a miR-7 target. In one motif (gray), Yan represses miR-7 directly and indirectly. In the indirect arm, Yan represses Phyllopod, alleviating repression of Ttk-69, which represses miR-7. In the second motif (red), Pnt-P1 directly activates $m i R-7$, which represses Yan. Pnt-P1 represses yan directly. (C) let-7 acts in a coherent feedforward motif (gray), modulating oncogenic transformation. $\mathrm{NF}-\mathrm{\kappa B}$ induces IL6 expression directly. In the indirect arm, NF-кB activity inhibits let-7, alleviating repression of IL6. let-7 prevents noise in the activity of NF- $\mathrm{BB}$ or IL6 from triggering the positive feedback loop (red). (D) miR-7 acts in an incoherent feedforward motif. Atonal activates E(spl) directly. Atonal also represses $\mathrm{E}(\mathrm{spl})$ via $\mathrm{miR}-7$. Atonal activity results in a pulse of E(spl) followed by a lower level of steady-state expression due to Atonal-induced $m i R-7$ activity. (E) $m i R-20$ and $m i R-17-5 p$ act in an incoherent feedforward motif. c-Myc activates E2F directly. c-Myc also represses E2F via $m i R-17-5 p$ and $m i R-20$. c-Myc activity results in a pulse of E2F to promote cell cycle progression.

that miRNAs work together with transcription factors to produce a switch that allows these cells to adopt one of two alternative fates, called ASEL/R (Fig. 1C). The homeobox-containing transcription factor encoded by $\operatorname{cog}-1$ is active in ASER, and the zinc finger transcription factor encoded by die-1 is active in ASEL. In ASEL cells, die-1 induces expression of the $1 s y-6$ miRNA, which has been shown to limit cog-1 activity in vivo. Reciprocally, in ASER, cog-1 induces expression of miR-273 family miRNAs, which are thought to act by repressing die-1. The asymmetry between the two cells is initially stabilized by positive autoregulation of cog-1 in ASER. The combination of miRNA-mediated repression and positive autoregulation produces a switch that drives the cell into one of two states. However, genetic evidence indicates that the two cells are initially identical. Both cells can adopt either ASER or ASEL identity if components of the feedback loops are missing. This initial state is labile, and is potentially subject to perturbation by noisy gene expression. A key issue that remains to be fully

understood is how an initial bias is introduced into the system to allow the switch to be thrown with a consistent left/right outcome.

miR-14 limits the impact of noise on a developmental switch

Drosophila miR-14 has been implicated in a different type of regulatory switch, which serves to buffer against noise (Varghese and Cohen 2007). The steroid hormone ecdysone and the ecdysone receptor $(\mathrm{EcR})$ regulate major transformations in gene expression programs as the animal transitions from one developmental stage to the next (for review, see Thummel 2001). An important feature of these transitions is their all-or-none character. This depends on a positive feedback loop involving transcriptional autoregulation by EcR. A loop in which a transcription factor controls its own expression is inherently labile. Transcription often occurs in bursts (Raj and van Oudenaarden 2008), which are expected to lead to fluctuation in activity levels. In principle, a transcription burst for an autoregulating transcription factor such as EcR could trigger a self-amplifying response. Linking EcR autoregulation to a negative feedback loop involving $m i R-14$ provides a means to limit the potential impact of noise (Fig. 1D). miR-14 acts directly to reduce EcR levels, and, reciprocally, EcR negatively regulates miR-14 transcription. Mutual repression keeps the levels of $\mathrm{miR}-14$ and EcR in balance, until the input of a hormonal cue activates EcR. This will shut off production of new $m i R-14$, but the preexisting miRNA takes time to decay. This delay permits the cell to discriminate between sustained input of hormone-induced EcR activation and transient fluctuation in EcR levels due to transcriptional bursts. The use of a miRNA to minimize the impact of noise permits the safe use of a positive autoregulatory loop in a switch capable of profoundly changing the gene expression program of the animal.

Repression of a repressor in a positive feedback loop: miR-9a

The regulatory network involving $m i R-9 a$ can also be seen as an indirect feedback loop. $m i R-9 a$ represses Senseless, which serves as an activator of the proneural genes. By repressing $m i R-9 a$, the proneural transcription factors remove repression of their activator, Senseless. Because the proneural genes promote Senseless expression, the net result is a positive autoregulatory loop (Fig. $1 \mathrm{~B})$. As in the case of $m i R-14$, the preexisting expression of the miRNA limits the impact of noise until it rises above a threshold level. Thereafter, the cell commits to a program of differentiation. An independent loop involving Delta and Notch conveys this information to influence the behavior of the other cells in the group.

Negative feedback in neuronal differentiation: miR-124 and ephrin-B1

Mutual inhibition of ephrin-B1 and miR-124 in the developing mouse brain illustrates a negative feedback 
interaction controlling neural differentiation (Arvanitis et al. 2010). Ephrins serve as both ligands and receptors that convey bidirectional signals reflecting cell-cell contact (for review, see Davy and Soriano 2005). Ephrin-B1 and $m i R-124$ show reciprocal expression during cortex differentiation. ephrin-B1 is expressed in neural progenitor layers, whereas $m i R-124$ expression is elevated in more differentiated cells. Ephrin-B1 signaling limits miR-124 levels, and, reciprocally, $m i R-124$ expression can inhibit ephrin-B1 expression. Thus, mutual inhibition between miR-124 and ephrin-B1 controls neuronal differentiation, where ephrin-B1 is required for maintaining the progenitor fate and miR-124 promotes differentiation (Fig. 1E). Mutual repression may help to sharpen expression boundaries and confer robustness during differentiation.

Negative feedback in epithelial-mesenchymal transition (EMT): the miR-200 family and ZEB/Sip1

EMT is a fundamental cellular transformation that contributes to shaping tissues during development. EMT also plays a key role during metastasis. Members of the miR200 miRNA family are expressed in epithelial cells, where they limit expression of the E-box transcription factors ZEB1 and SIP1, thereby allowing expression of E-Cadherin (Gregory et al. 2008; Park et al. 2008). ZEB1 and SIP1 are induced by TGF- $\beta$ to promote EMT though repression of E-Cadherin. ZEB1 and SIP1 also repress expression of the miR-200 family of miRNAs (Bracken et al. 2008). The mutually repressive regulatory relationship is similar to that between miR-124 and Ephrin-B1. Mutual inhibition helps to reinforce the switch between cell fates, and may help to ensure that low-level noisy expression of ZEB1 or SIP1 cannot accidentally trigger EMT prior to the onset of robust TGF- $\beta$-induced expression. During EMT, the cells do not remain together as part of a tissue, so the reciprocity may be thought of as being more temporal than spatial.

\section{A miRNA-regulated feedback loop in glucose homeostasis}

Energy homeostasis depends on insulin signaling. Insulin levels reflect the nutritional status of the animal to control circulating sugar levels and to regulate glycogen and fat metabolism. Insulin production and secretion as well as cellular responsiveness to insulin are subject to regulation by miRNAs. miRNAs have been shown to act in many tissues involved in glucose metabolism, including the pancreatic islet, liver, skeletal muscle, adipose tissue, and brain (for review, see Lynn 2009). In vivo, mice mutant for $m i R-375$ show reduced pancreatic $\beta$-cell mass and defects in energy homeostasis (Poy et al. 2009). In assays using insulin-producing cell lines, $m i R-375$ has been shown to negatively regulate glucose-stimulated insulin release (Poy et al. 2004). miR-375 has been reported to act via its target myotrophin to control a late step during insulin exocytosis. Although the mechanism is not fully understood, it probably involves regulation of actin polymerization and vesicular fusion. miRNAs are also thought to regulate insulin levels by other means.
Pdpk1, a 3'-phosphoinositoside-dependent protein kinase, is a direct target of $m i R-375$. Depletion of $m i R-375$ has been reported to increase Pdpk-1 levels and to increase insulin expression, also in a cell-based assay system (El Ouaamari et al. 2008). Intriguingly, $m i R-375$ expression appears to be regulated by glucose levels in rat pancreatic islets. Thus, glucose sensing appears to act via repression of $\mathrm{miR}-375$ to promote insulin secretion, contributing to glucose homeostasis (Fig. 1F).

\section{Managing noise: coherent feedforward motifs as buffers}

Feedforward motifs involve at least three elements: an upstream regulator and two targets (illustrated in Fig. 2A; for review, see Alon 2007). The upstream element X acts on one target, $Z$, directly as well as indirectly, via the second target, Y. In cases in which both branches act in the same direction on $\mathrm{Z}$, the motif is called "coherent." If they act oppositely, the motif is called "incoherent." Motifs of this sort can have many possible outcomes, depending on the nature of the relationships between the elements. For example, a coherent motif in which $X$ and $\mathrm{Y}$ are both needed to activate $\mathrm{Z}$ introduces a delay in the induction of $\mathrm{Z}$ until $\mathrm{Y}$ can accumulate to the required level. A coherent motif in which either $\mathrm{X}$ or $\mathrm{Y}$ can activate $Z$ allows for rapid onset of $Z$ but delays turning $Z$ off, to an extent that depends on the stability of $Y$. Incoherent motifs have different properties; for example, they can allow for rapid onset followed by repression to produce a pulse-like activation profile. Alon (2007) provides an in-depth review of the properties of these motifs. In the following sections, we focus on examples that involve miRNAs.

\section{Coherent feedforward motifs in photoreceptor differentiation}

miR-7 has been implicated in two coherent feedforward motifs involved in the specification of photoreceptor cells in the Drosophila eye (Fig. 2B; Li et al. 2009). Yan, a transcription factor essential for the development photoreceptor cells, is a direct target of $m i R-7$. In one circuit, Yan represses $m i R-7$ transcription directly as well as indirectly (Fig. 2B, shown in gray). The indirect arm in this case involves three elements: Yan represses Phyllopod, thereby alleviating repression of Ttk-69, which allows Ttk-69 to repress $\mathrm{miR}-7$ (the triple negative, repression of a repressor of a repressor results in repression). Repression by either branch is sufficient to limit $m i R-7$ expression. This makes the system less sensitive to transient fluctuation in the levels of either Yan or Ttk-69. Concurrently, $m i R-7$ is involved in a second coherent feedforward motif in the same cells. The transcription factor Pnt-P1 directly activates miR-7 transcription, which in turn represses Yan. Pnt-P1 also acts directly to represses yan transcription. This circuit buffers Yan expression to variations in the levels of Pnt-P1 or miR-7. The Yan OFF state is buffered against transient fluctuation in Pnt-P1 levels, but can switch if activation is prolonged. Likewise the Yan ON state is stable. It is likely that use of two 
interlinked motifs with elements in common provides considerable stability to photoreceptor specification. Indeed, Li et al. (2009) provide experimental evidence that the system is normally very stable, but that it can be perturbed by environmental fluctuation-temperature shifts in this instance-if miR-7 function is compromised.

\section{A coherent feedforward motif limits the impact of noise in an oncogenic switch}

A recent study has implicated the human let-7 miRNA in a molecular pathway underlying oncogenic transformation in response to an inflammatory signal (Iliopoulos et al. 2009). Loss of let-7 has been implicated previously in cancer (e.g., Johnson et al. 2005). let-7 activity normally serves to limit expression of the cytokine interleukin-6 (IL6), a key mediator of the inflammatory response. Activation of NF- $\mathrm{kB}$ can block let-7 family miRNA biogenesis through up-regulation of lin-28 (which prevents let-7 processing) (Viswanathan et al. 2008). Sustained NF- $\kappa \mathrm{B}$ activation thereby leads to depletion of let-7 and alleviates repression of IL6. This comprises the indirect arm of the feedforward motif (Fig. 2C, gray). NF-кB also acts directly to induce IL6 expression. Activation of both arms of the feedforward motif is required to achieve the high levels of IL6 activity required for cellular transformation (Iliopoulos et al. 2009), which is aided by positive feedback regulation of IL6 on NF-kB (Fig. 2C, red). In essence, the role of let-7 is comparable with that of miR-14 in the steroid hormone switch described above. By limiting levels of IL6, let-7 prevents noise in the activity of NF- $\mathrm{KB}$ or IL6 from triggering the positive feedback loop that leads to cellular transformation. Only sustained activation of NF- $\mathrm{kB}$ can allow the decay of miRNA expression required for the IL6/NF-kB feedback loop to become self-sustaining. Comparing let-7 and miR-14 illustrates how similar biological outputs can be obtained by regulatory networks of different topology.

\section{Incoherent feedforward motifs as triggers}

The Drosophila sensory nervous system is proving to be a rich source of feedforward regulatory motifs. Work from the Carthew laboratory (Li et al. 2009) has also implicated miR-7 in an incoherent feedforward motif that contributes to sense organ specification. In this context, the transcription factor Atonal acts directly to activate expression of the proneural gene $\mathrm{E}(\mathrm{spl})$, illustrated in Figure 2D. Atonal acts concurrently via miR-7 to repress $E(\mathrm{spl})$ genes. Activation of Atonal results in a pulse of $\mathrm{E}(\mathrm{spl})$ expression followed by a lower level of steady-state expression. The pulse of $\mathrm{E}(\mathrm{Spl})$ can serve as a trigger for other regulatory events, including those involving $m i R-9 a$ and Senseless.

A second example of an incoherent regulatory motif involves two miRNAs of the human miR-17 cluster in regulating cell cycle progression (Fig. 2E; O'Donnell et al. 2005). c-Myc is a potent inducer of cell proliferation, and is sufficient to promote tumor formation (Pelengaris et al. 2002). c-Myc acts directly to induce expression of the transcription factor $E 2 F$, which in turn regulates an array of cell cycle genes. c-Myc acts concurrently to induce expression of $m i R-17-5 p$ and $m i R-20$, which then repress E2F. This leads to a pulse of c-Myc-induced E2F expression to promote cell cycle progression. Incoherent feedforward motifs are well suited as triggers for oscillatory systems. A potentially important implication of the use of such motifs is that the output can be shorter-lived than the input.

\section{Conclusions}

The studies discussed here represent only a small fraction of what has been learned in recent years about the roles of miRNAs in vivo. They represent the cases in which it has been possible to place the miRNA into a gene regulatory circuit, and from this it has been possibe to understand how the miRNA confers a specific mode of biological regulation. These circuits can have quite different roles, from using noise to throw a developmental switch to buffering the consequences of noise in order to confer robustness to environmental perturbation.

\section{Acknowledgments}

We thank Ruifen Weng, Xin Hong, Jan-Michael Kugler, and Jishy Varghese for discussions and comments on the manuscript.

\section{References}

Alon U. 2007. Network motifs: Theory and experimental approaches. Nat Rev Genet 8: 450-461.

Arvanitis DN, Jungas T, Behar A, Davy A. 2010. Ephrin-B1 reverse signaling controls a post-transcriptional feedback mechanism via miR-124. Mol Cell Biol 30: 2508-2517.

Barkai N, Shilo BZ. 2007. Variability and robustness in biomolecular systems. Mol Cell 28: 755-760.

Bartel DP. 2009. MicroRNAs: Target recognition and regulatory functions. Cell 136: 215-233.

Bracken CP, Gregory PA, Kolesnikoff N, Bert AG, Wang J, Shannon MF, Goodall GJ. 2008. A double-negative feedback loop between ZEB1-SIP1 and the microRNA-200 family regulates epithelial-mesenchymal transition. Cancer Res 68: 7846-7854.

Bushati N, Cohen SM. 2007. MicroRNA functions. Annu Rev Cell Dev Biol 23: 175-205.

Cohen SM, Brennecke J, Stark A. 2006. Denoising feedback loops by thresholding-a new role for microRNAs. Genes Dev 20: $2769-2772$.

Davy A, Soriano P. 2005. Ephrin signaling in vivo: Look both ways. Dev Dyn 232: 1-10.

El Ouaamari A, Baroukh N, Martens GA, Lebrun P, Pipeleers D, van Obberghen E. 2008. miR-375 targets 3'-phosphoinositidedependent protein kinase-1 and regulates glucose-induced biological responses in pancreatic $\beta$-cells. Diabetes 57: 2708-2717.

Eulalio A, Huntzinger E, Izaurralde E. 2008. Getting to the root of miRNA-mediated gene silencing. Cell 132: 9-14.

Gregory PA, Bert AG, Paterson EL, Barry SC, Tsykin A, Farshid G, Vadas MA, Khew-Goodall Y, Goodall GJ. 2008. The miR-200 family and miR-205 regulate epithelial to mesenchymal transition by targeting ZEB1 and SIP1. Nat Cell Biol 10: 593-601.

Hobert O. 2006. Architecture of a microRNA-controlled gene regulatory network that diversifies neuronal cell fates. Cold Spring Harb Symp Quant Biol 71: 181-188. 
Iliopoulos D, Hirsch HA, Struhl K. 2009. An epigenetic switch involving NF- $\mathrm{B}$, Lin28, Let-7 microRNA, and IL6 links inflammation to cell transformation. Cell 139: 693-706.

Jafar-Nejad H, Acar M, Nolo R, Lacin H, Pan H, Parkhurst SM, Bellen HJ. 2003. Senseless acts as a binary switch during sensory organ precursor selection. Genes Dev 17: 2966-2978.

Johnson SM, Grosshans H, Shingara J, Byrom M, Jarvis R, Cheng A, Labourier E, Reinert KL, Brown D, Slack FJ. 2005. RAS is regulated by the let-7 microRNA family. Cell 120: 635647.

Lee TI, Rinaldi NJ, Robert F, Odom DT, Bar-Joseph Z, Gerber GK, Hannett NM, Harbison CT, Thompson CM, Simon I, et al. 2002. Transcriptional regulatory networks in Saccharomyces cerevisiae. Science 298: 799-804.

Li Y, Wang F, Lee JA, Gao FB. 2006. MicroRNA-9a ensures the precise specification of sensory organ precursors in Drosophila. Genes Dev 20: 2793-2805.

Li X, Cassidy JJ, Reinke CA, Fischboeck S, Carthew RW. 2009. A microRNA imparts robustness against environmental fluctuation during development. Cell 137: 273-282.

Losick R, Desplan C. 2008. Stochasticity and cell fate. Science 320: $65-68$.

Lynn FC. 2009. Meta-regulation: MicroRNA regulation of glucose and lipid metabolism. Trends Endocrinol Metab 20: 452-459.

Martinez NJ, Ow MC, Barrasa MI, Hammell M, Sequerra R, Doucette-Stamm L, Roth FP, Ambros VR, Walhout AJ. 2008. A C. elegans genome-scale microRNA network contains composite feedback motifs with high flux capacity. Genes Dev 22: 2535-2549.

Milo R, Shen-Orr S, Itzkovitz S, Kashtan N, Chklovskii D, Alon U. 2002. Network motifs: Simple building blocks of complex networks. Science 298: 824-827.

Nolo R, Abbott LA, Bellen HJ. 2000. Senseless, a Zn finger transcription factor, is necessary and sufficient for sensory organ development in Drosophila. Cell 102: 349-362.

O'Donnell KA, Wentzel EA, Zeller KI, Dang CV, Mendell JT. 2005. c-Myc-regulated microRNAs modulate E2F1 expression. Nature 435: 839-843.

Park SM, Gaur AB, Lengyel E, Peter ME. 2008. The miR-200 family determines the epithelial phenotype of cancer cells by targeting the E-cadherin repressors ZEB1 and ZEB2. Genes Dev 22: 894-907.

Pelengaris S, Khan M, Evan GI. 2002. Suppression of Mycinduced apoptosis in $\beta$ cells exposes multiple oncogenic properties of Myc and triggers carcinogenic progression. Cell 109: 321-334.

Poy MN, Eliasson L, Krutzfeldt J, Kuwajima S, Ma X, Macdonald PE, Pfeffer S, Tuschl T, Rajewsky N, Rorsman P, et al. 2004. A pancreatic islet-specific microRNA regulates insulin secretion. Nature 432: 226-230.

Poy MN, Hausser J, Trajkovski M, Braun M, Collins S, Rorsman P, Zavolan M, Stoffel M. 2009. miR-375 maintains normal pancreatic $\alpha$ - and $\beta$-cell mass. Proc Natl Acad Sci 106: 58135818.

Raj A, van Oudenaarden A. 2008. Nature, nurture, or chance: Stochastic gene expression and its consequences. Cell 135: 216-226.

Raser JM, O'Shea EK. 2005. Noise in gene expression: Origins, consequences, and control. Science 309: 2010-2013.

Stark A, Brennecke J, Bushati N, Russell RB, Cohen SM. 2005. Animal microRNAs confer robustness to gene expression and have a significant impact on 3'UTR evolution. Cell 123: 1133-1146.

Stefani G, Slack FJ. 2008. Small non-coding RNAs in animal development. Nat Rev Mol Cell Biol 9: 219-230.
Thummel CS. 2001. Molecular mechanisms of developmental timing in C. elegans and Drosophila. Dev Cell 1: 453-465.

Tsang J, Zhu J, van Oudenaarden A. 2007. MicroRNA-mediated feedback and feedforward loops are recurrent network motifs in mammals. Mol Cell 26: 753-767.

Varghese J, Cohen SM. 2007. MicroRNA miR-14 acts to modulate a positive autoregulatory loop controlling steroid hormone signaling in Drosophila. Genes Dev 21: 2277-2282.

Viswanathan SR, Daley GQ, Gregory RI. 2008. Selective blockade of microRNA processing by Lin28. Science 320: 97-100. 


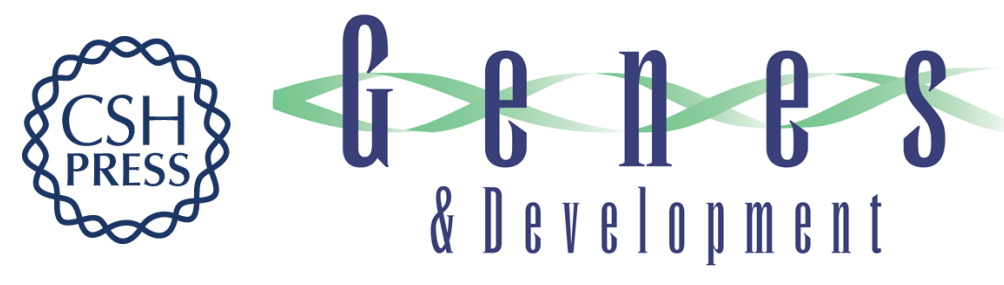

\section{MicroRNAs and gene regulatory networks: managing the impact of noise in biological systems}

Héctor Herranz and Stephen M. Cohen

Genes Dev. 2010, 24:

Access the most recent version at doi:10.1101/gad.1937010

References This article cites 36 articles, 16 of which can be accessed free at: http://genesdev.cshlp.org/content/24/13/1339.full.html\#ref-list-1

License

Email Alerting

Receive free email alerts when new articles cite this article - sign up in the box at the top Service right corner of the article or click here.

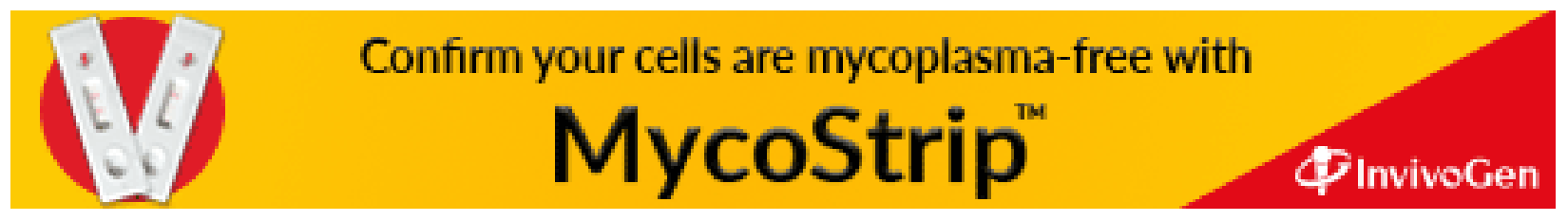

